\title{
Quantum Mechanical Calculations of NMR J-Coupling Values in the Determination of Relative Configuration in Organic Compounds
}

Giuseppe Bifulco, Carla Bassarello, Raffaele Riccio and Luigi Gomez-Paloma*

Dipartimento di Scienze Farmaceutiche, Università di Salerno, 84084 Fisciano (SA), Italy

gomez@unisa.it

Supporting Information Available

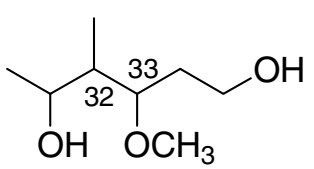

C32-C33

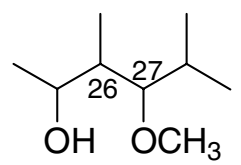

C26-C27

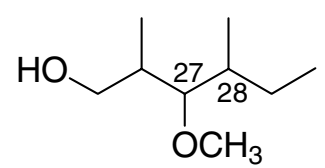

C27-C28

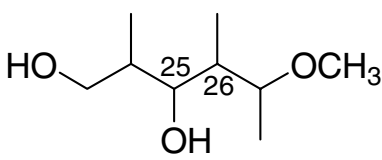

$\mathrm{C} 25-\mathrm{C} 26$<smiles>COCC(C)C(O)[14C](C)CO</smiles>

C24-C25 
Table S1. Experimental and calculated $J$ values of the entire molecule 2 (only the relevant 3D arrangements are considered, see also Table 2)

\begin{tabular}{|c|c|c|c|c|}
\hline $\begin{array}{l}{ }_{3} J_{\mathrm{H} 32-\mathrm{H} 33} \\
{ }^{2} J_{\text {H32-C33 }} \\
{ }^{3} J_{\text {H32-C34 }} \\
3_{3} J_{\mathrm{H} 3-\mathrm{C} 31} \\
{ }^{3} J_{\text {H33-Me32 }}\end{array}$ & $\begin{array}{c}\text { exp. } \\
\mathrm{g}^{+} \text {erythro } \\
2.6 \\
-5.4 \\
4.8 \\
0.5 \\
4.4\end{array}$ & $\begin{array}{c}\text { C32-C33 } \\
\text { calc. } \\
\mathrm{g}^{+} \text {erythro } \\
2.8 \\
-4.4 \\
5.0 \\
0.9 \\
4.4\end{array}$ & 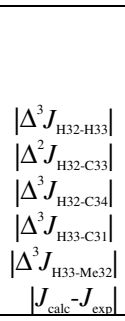 & $\begin{array}{l}0.2 \\
1.0 \\
0.2 \\
0.4 \\
0.0 \\
\mathbf{1 . 8}\end{array}$ \\
\hline $\begin{array}{l}{ }_{3} J_{\text {H27-H28 }} \\
{ }^{2} J_{\text {H28-C27 }} \\
{ }^{3} J_{\text {H28-CH26 }} \\
{ }^{3} J_{\text {H27-CH29 }} \\
{ }^{3} J_{\text {H27-Me28 }}\end{array}$ & $\begin{array}{c}\text { exp. } \\
\text { anti erythro } \\
8.9 \\
-3.6 \\
1.0 \\
2.2 \\
1.4\end{array}$ & $\begin{array}{c}\text { C27-C28 } \\
\text { calc. } \\
\text { anti erythro } \\
8.6 \\
-3.7 \\
1.1 \\
3.5 \\
1.0\end{array}$ & $\begin{array}{r}\left|\Delta^{3} J_{\text {H27-- } 28}\right| \\
\left|\Delta^{2} J_{\text {H28-C27 }}\right| \\
\left|\Delta^{3} J_{\text {H28-CH26 }}\right| \\
\left|\Delta^{3} J_{\text {H27-CH29 }}\right| \\
\left|\Delta^{3} J_{\text {H27-Me28 }}\right| \\
\left|J_{\text {calc }} J_{\text {exp }}\right|\end{array}$ & $\begin{array}{l}0.3 \\
0.1 \\
0.1 \\
1.3 \\
0.4 \\
\mathbf{2 . 2}\end{array}$ \\
\hline $\begin{array}{l}{ }^{3} J_{\text {H26-H27 }} \\
{ }^{2} J_{\text {H26-C27 }} \\
{ }^{3} J_{\text {H26-C28 }} \\
3^{3} J_{\text {H27-C25 }} \\
{ }^{3} J_{\text {H27-Me26 }}\end{array}$ & $\begin{array}{c}\text { exp. } \\
\text { g erythro } \\
2.4 \\
-1.5 \\
0.5 \\
6.7 \\
3.1\end{array}$ & $\begin{array}{c}\text { C26-C27 } \\
\text { calc. } \\
\text { g erythro } \\
2.2 \\
0.5 \\
0.3 \\
5.0 \\
3.1\end{array}$ & $\begin{array}{r}\left|\Delta^{3} J_{\text {H26-H27 }}\right| \\
\left|\Delta^{2} J_{\text {H26-C27 }}\right| \\
\left|\Delta^{3} J_{\text {H26-C28 }}\right| \\
\left|\Delta^{3} J_{\text {H27-C25 }}\right| \\
\left|\Delta^{3} J_{\text {H27-Me26 }}\right| \\
\left|J_{\text {calc }} J_{\text {exx }}\right|\end{array}$ & $\begin{array}{l}0.2 \\
2.0 \\
0.2 \\
1.7 \\
0.0 \\
\mathbf{4 . 1}\end{array}$ \\
\hline $\begin{array}{l}{ }^{3} J_{\mathrm{H} 25-\mathrm{H} 26} \\
{ }^{2} J_{\mathrm{H} 26-\mathrm{C} 25} \\
{ }^{3} J_{\mathrm{H} 26-\mathrm{C} 24} \\
{ }^{3} J_{\mathrm{H} 25-\mathrm{C} 27} \\
{ }^{3} J_{\mathrm{H} 25-\mathrm{Me} 26}\end{array}$ & $\begin{array}{c}\text { exp. } \\
\mathrm{g}^{+} \text {threo } \\
1.2 \\
-0.5 \\
0.5 \\
0.9 \\
6.7\end{array}$ & $\begin{array}{c}\text { C25-C26 } \\
\text { calc. } \\
\mathrm{g}^{+} \text {threo } \\
1.5 \\
-0.5 \\
0.8 \\
2.3 \\
4.2\end{array}$ & $\begin{array}{r}\left|\Delta^{3} J_{\text {H25-H26 }}\right| \\
\left|\Delta^{2} J_{\text {H26-C25 }}\right| \\
\left|\Delta^{3} J_{\text {H26-C24 }}\right| \\
\left|\Delta^{3} J_{\text {H25-C27 }}\right| \\
\left|\Delta^{3} J_{\text {H25-Me26 }}\right| \\
\left|J_{\text {calc }} J_{\text {exx }}\right|\end{array}$ & $\begin{array}{l}0.3 \\
0.0 \\
0.3 \\
1.4 \\
2.5 \\
\mathbf{4 . 5}\end{array}$ \\
\hline $\begin{array}{l}{ }^{3} J_{\mathrm{H} 24-\mathrm{H} 25} \\
{ }^{2} J_{\mathrm{H} 24-\mathrm{C} 25} \\
{ }^{3} J_{\mathrm{H} 24 \mathrm{-} 26} \\
{ }^{3} J_{\mathrm{H} 25-\mathrm{C} 23} \\
{ }^{3} J_{\mathrm{H}-\mathrm{-M} 24}\end{array}$ & $\begin{array}{c}\text { exp. } \\
\text { anti erythro } \\
9.9 \\
-2.4 \\
1.1 \\
3.3 \\
1.4\end{array}$ & $\begin{array}{c}\text { C24-C25 } \\
\text { calc. } \\
\text { anti erythro } \\
8.2 \\
-3.5 \\
1.4 \\
3.5 \\
1.2\end{array}$ & 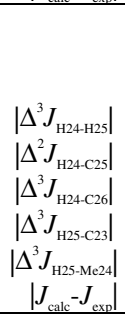 & $\begin{array}{l}1.7 \\
1.1 \\
0.3 \\
0.2 \\
0.2 \\
\mathbf{3 . 5}\end{array}$ \\
\hline
\end{tabular}


Table S2. Calculated $J$-values for the entire molecule $\mathbf{2}$ and for its $\mathrm{C}_{2}$ reduced fragments.

\begin{tabular}{|c|c|c|c|c|}
\hline $\begin{array}{l}{ }^{3} J_{\mathrm{H} 2-\mathrm{H} 33} \\
{ }^{2} J_{332-\mathrm{C} 33} \\
{ }^{3} J_{\mathrm{H} 2-\mathrm{C} 34} \\
{ }^{3} J_{\mathrm{H} 3-\mathrm{C} 31} \\
{ }^{3} J_{\mathrm{H} 33-\mathrm{M} 32}\end{array}$ & $\begin{array}{c}\text { entire molecule } \\
\mathrm{g}^{+} \text {erythro } \\
2.8 \\
-4.4 \\
5.0 \\
0.9 \\
4.4\end{array}$ & $\begin{array}{c}\text { C32-C33 } \\
\text { single arrangement } \\
\text { g }^{+} \text {erythro } \\
2.4 \\
-4.7 \\
4.4 \\
1.9 \\
4.8\end{array}$ & $\begin{array}{l}\left|\Delta^{3} J_{\mathrm{H} 2-\mathrm{H} 33}\right| \\
\left|\Delta^{2} J_{\mathrm{H} 2-\mathrm{C} 33}\right| \\
\left|\Delta^{3} J_{\text {H32-C34 }}\right| \\
\left|\Delta^{3} J_{\text {H33-C31 }}\right| \\
\left|\Delta^{3} J_{\text {H33-M } 32}\right| \\
\Sigma\left|J_{\text {calc }} J_{\text {exp }}\right|\end{array}$ & $\begin{array}{l}0.4 \\
0.3 \\
0.6 \\
1.0 \\
0.4 \\
\mathbf{2 . 7}\end{array}$ \\
\hline $\begin{array}{l}{ }^{3} J_{\text {H27-H28 }} \\
{ }^{2} J_{\text {H28-C27 }} \\
{ }^{3} J_{\text {H28-CH26 }} \\
{ }^{3} J_{\text {H27-CH29 }} \\
{ }^{3} J_{\text {H27-Me28 }}\end{array}$ & $\begin{array}{c}\text { entire molecule } \\
\text { anti erythro } \\
8.6 \\
-3.7 \\
1.1 \\
3.5 \\
1.0\end{array}$ & $\begin{array}{c}\text { C27-C28 } \\
\text { single arrangement } \\
\text { anti erythro } \\
8.6 \\
-3.5 \\
1.6 \\
2.7 \\
1.9\end{array}$ & $\begin{array}{l}\left|\Delta^{3} J_{\mathrm{H} 27-\mathrm{H} 28}\right| \\
\left|\Delta^{2} J_{\mathrm{H} 28-\mathrm{C} 27}\right| \\
\left|\Delta^{3} J_{\mathrm{H} 28-\mathrm{CH} 26}\right| \\
\left|\Delta^{3} J_{\mathrm{H} 27-\mathrm{CH} 2}\right| \\
\left|\Delta^{3} J_{\text {H27-Me28 }}\right| \\
\Sigma\left|J_{\text {calc }} J_{\text {exp }}\right|\end{array}$ & $\begin{array}{l}0.0 \\
0.2 \\
0.5 \\
0.8 \\
0.9 \\
\mathbf{2 . 4}\end{array}$ \\
\hline $\begin{array}{l}{ }^{3} J_{\mathrm{H} 26-\mathrm{H} 27} \\
{ }^{2} J_{\mathrm{H} 26-\mathrm{C} 27} \\
{ }^{3} J_{\mathrm{H} 26-\mathrm{C} 28} \\
{ }^{3} J_{\mathrm{H}-\mathrm{C} 25} \\
{ }^{3} J_{\mathrm{H} 27-\mathrm{Me} 26}\end{array}$ & $\begin{array}{c}\text { entire molecule } \\
\text { g erythro } \\
2.2 \\
0.5 \\
0.3 \\
5.0 \\
3.1\end{array}$ & $\begin{array}{c}\text { C26-C27 } \\
\text { single arrangement } \\
\text { g erythro } \\
1.9 \\
0.9 \\
0.4 \\
5.0 \\
3.3\end{array}$ & $\begin{array}{l}\left|\Delta^{3} J_{\text {H26-H27 }}\right| \\
\left|\Delta^{2} J_{\text {H26-C27 }}\right| \\
\left|\Delta^{3} J_{\text {H26-C28 }}\right| \\
\left|\Delta^{3} J_{\text {H27-C25 }}\right| \\
\left|\Delta^{3} J_{\text {H27-Me26 }}\right| \\
\Sigma\left|J_{\text {call }} J_{\text {exx }}\right|\end{array}$ & $\begin{array}{l}0.3 \\
0.4 \\
0.1 \\
0.0 \\
0.2 \\
\mathbf{1 . 0}\end{array}$ \\
\hline $\begin{array}{l}{ }^{3} J_{\mathrm{H} 25-\mathrm{H} 26} \\
{ }^{2} J_{\mathrm{H} 26-\mathrm{C} 25} \\
{ }^{3} J_{\mathrm{H} 26-\mathrm{C} 24} \\
{ }^{3} J_{\text {H25-C27 }} \\
{ }^{3} J_{\text {H25-Me26 }}\end{array}$ & $\begin{array}{c}\text { entire molecule } \\
\mathrm{g}^{+} \text {threo } \\
1.5 \\
-0.5 \\
0.8 \\
2.3 \\
4.2\end{array}$ & $\begin{array}{c}\text { C25-C26 } \\
\text { single arrangement } \\
\mathrm{g}^{+} \text {threo } \\
3.6 \\
0.8 \\
0.7 \\
1.3 \\
5.7\end{array}$ & $\begin{array}{l}\left|\Delta^{3} J_{\text {H25-H22 }}\right| \\
\left|\Delta^{2} J_{\text {H26-C25 }}\right| \\
\left|\Delta^{3} J_{\text {H26-C24 } 2}\right| \\
\left|\Delta^{3} J_{\text {H25-C27 }}\right| \\
\left|\Delta^{3} J_{\text {H25-Me26 }}\right| \\
\Sigma\left|J_{\text {calc }}-J_{\text {exp }}\right|\end{array}$ & $\begin{array}{l}2.1 \\
1.3 \\
0.1 \\
1.0 \\
1.5 \\
6.0\end{array}$ \\
\hline $\begin{array}{l}{ }^{3} J_{\mathrm{H} 24-\mathrm{H} 25} \\
{ }^{2} J_{\text {H24-C25 }} \\
{ }^{3} J_{\text {H24-C26 }} \\
{ }^{3} J_{\text {H25-C23 }} \\
{ }^{3} J_{\text {H25-Me24 }}\end{array}$ & $\begin{array}{c}\text { entire molecule } \\
\text { anti erythro } \\
8.2 \\
-3.5 \\
1.4 \\
3.5 \\
1.2\end{array}$ & $\begin{array}{c}\text { C24-C25 } \\
\text { single arrangement } \\
\text { anti erythro } \\
8.6 \\
-3.7 \\
1.3 \\
3.4 \\
1.2\end{array}$ & $\begin{array}{l}\left|\Delta^{3} J_{\mathrm{H} 24-\mathrm{H} 25}\right| \\
\left|\Delta^{2} J_{\mathrm{H} 24-\mathrm{C} 25}\right| \\
\left|\Delta^{3} J_{\mathrm{H} 24-\mathrm{C} 26}\right| \\
\left|\Delta^{3} J_{\mathrm{H} 25-\mathrm{C} 23}\right| \\
\left|\Delta^{3} J_{\mathrm{H} 25-\mathrm{Me} 24}\right| \\
\Sigma\left|J_{\text {calc }}-J_{\text {exp }}\right|\end{array}$ & $\begin{array}{l}0.4 \\
0.2 \\
0.1 \\
0.1 \\
0.0 \\
\mathbf{0 . 8}\end{array}$ \\
\hline
\end{tabular}


Table S3. Absolute differences $|\Delta J|$ between the experimental $J$-coupling values and the corresponding calculated values found for the entire molecule 2 and for its reduced $\mathrm{C}_{2}$ fragments, respectively.

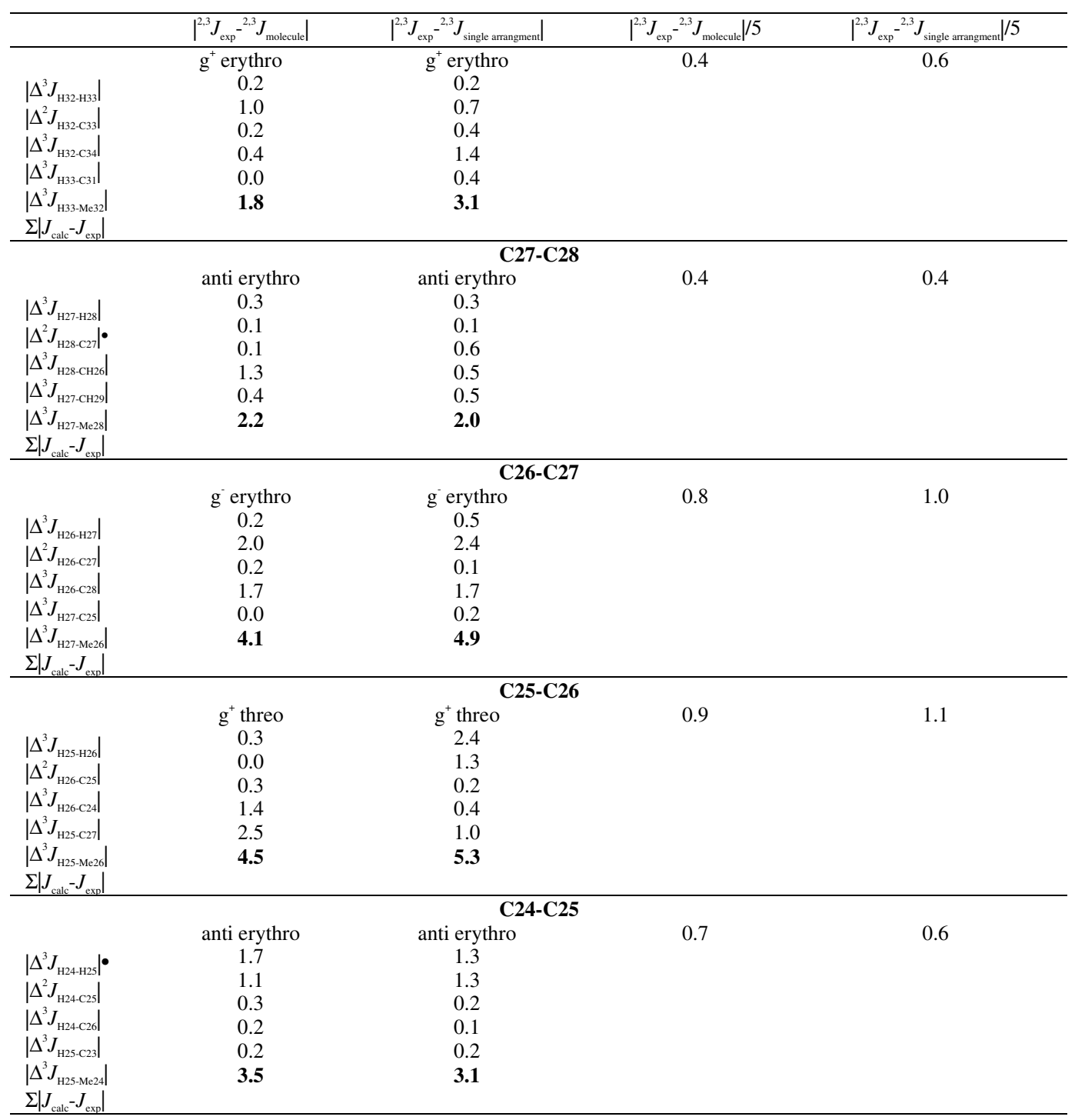


Table S4. Experimental and calculated (total coupling and Fermi contact, spin-dipole and spin-orbit terms) $J$-values for the $\mathrm{C}_{2}$ reduced fragments.

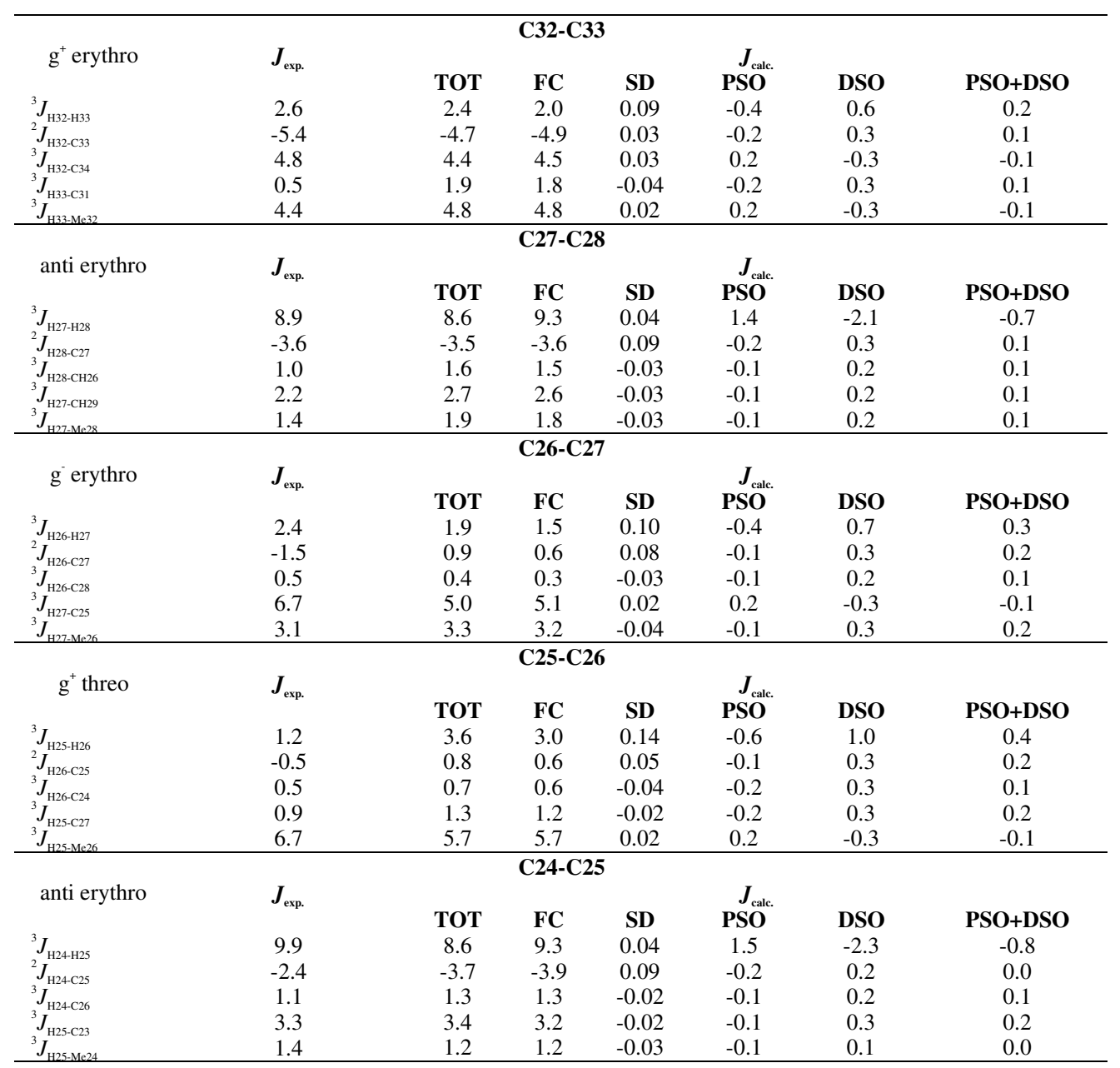




\section{NMR Spectroscopy}

NMR measurements were performed on a Bruker DRX-600 spectrometer at $300 \mathrm{~K}$. All spectra were acquired in the phase-sensitive mode and the TPPI method was used for quadrature detection in the $\omega_{1}$ dimension. The molecule 2 was prepared dissolving $2.8 \mathrm{mg}$ in $0.5 \mathrm{~mL}$ of $\mathrm{CDCl}_{3}$ (Carlo Erba, 99.95\% D). ${ }^{3} J_{\mathrm{H}-\mathrm{H}}$ values were extracted from $1 \mathrm{D}{ }^{1} \mathrm{H} \mathrm{NMR}$ and 2D P.E.COSY spectra. For the P.E.COSY spectrum 128 scans per $\mathrm{t}_{1}$ value were acquired with a $\mathrm{t}_{1 \max }$ of $39 \mathrm{~ms} .{ }^{2,3} J_{\text {C-H }}$ values were obtained from phase-sensitive PFG-HETLOC and phase-sensitive PFG-PS-HMBC spectra according the following conditions. For the PFG-HETLOC spectrum, a total of $196 \mathrm{scans} / \mathrm{t}_{1}$ were acquired using $4 \mathrm{~K}$ points in $\omega_{2}$, with a spin lock of $50 \mathrm{~ms}$ and a $\mathrm{t}_{1 \max }$ of $53.8 \mathrm{~ms}$. The data matrix was zero-filled to $8 \mathrm{Kx} 1 \mathrm{~K}$ affording a digital resolution of $0.5 \mathrm{~Hz}$ in $\omega_{2}$. The PFG-PS-HMBC spectrum was recorded using $4 \mathrm{~K}$ points in $\omega_{2}$, setting the delay for long-range coupling evolution $(\Delta)$ at 50 $\mathrm{ms}$, with $128 \mathrm{scans} / \mathrm{t}_{1}\left(\mathrm{t}_{\operatorname{lmax}} 12.2 \mathrm{~ms}\right)$. Zero-filling $(8 \mathrm{Kx} 1 \mathrm{~K})$ was carried out in $\omega_{2}$ and $\omega_{1}$, respectively, to obtain a digital resolution of $0.3 \mathrm{~Hz}$ in $\omega_{2}$. The NMR data were processed on a Silicon Graphic Indigo2 workstation using the Bruker XWIN-NMR software.

For the QM calculations, both the full geometry optimization and the calculation of $J$-coupling values were performed on a Pentium-4 at $2.8 \mathrm{GHz}$ processor (running Microsoft Windows 2000) using the Gaussian03W program package. The entire molecule 2 and the gauche or anti staggered conformers of the five simplified fragments (Figure S1) were optimised at mPW1PW91 level of theory using the 6-31G(d) basis set; the calculation of $J$-coupling was executed on the optimized geometries using the same mPW1PW91 functional and the 6-31G(d,p) basis set. For the latter calculation the PCM solvent continuum model was used. Geometry optimization executed on the staggered arrangements was performed first at SCF level using the AM1 halmitionian and then at DFT level. 